\title{
Characteristics of compressible flow of supercritical kerosene
}

\author{
Feng-Quan Zhong • Xue-Jun Fan • Jing Wang • Gong Yu • Jian-Guo Li
}

Received: 2 March 2010 / Revised: 25 May 2011 / Accepted: 22 June 2011

(C)The Chinese Society of Theoretical and Applied Mechanics and Springer-Verlag Berlin Heidelberg 2012

\begin{abstract}
In this paper, compressible flow of aviation kerosene at supercritical conditions has been studied both numerically and experimentally. The thermophysical properties of supercritical kerosene are calculated using a 10species surrogate based on the principle of extended corresponding states (ECS). Isentropic acceleration of supercritical kerosene to subsonic and supersonic speeds has been analyzed numerically. It has been found that the isentropic relationships of supercritical kerosene are significantly different from those of ideal gases. A two-stage fuel heating and delivery system is used to heat the kerosene up to a temperature of $820 \mathrm{~K}$ and pressure of $5.5 \mathrm{MPa}$ with a maximum mass flow rate of $100 \mathrm{~g} / \mathrm{s}$. The characteristics of supercritical kerosene flows in a converging-diverging nozzle (Laval nozzle) have been studied experimentally. The results show that stable supersonic flows of kerosene could be established in the temperature range of $730 \mathrm{~K}-820 \mathrm{~K}$ and the measurements in the wall pressure agree with the numerical calculation.
\end{abstract}

Keywords Supercritical kerosene - Compressible flow . Laval nozzle $\cdot$ Isentropic process

\section{Introduction}

Hydrocarbon fuels have been used for the regenerative cooling of a scramjet engine [1-3]. Before entering the combustor, the fuel state varies with different operation conditions. In the early stage (low-speed), because the amount of heat

The project was supported by the National Natural Science Foundation of China (10672169 and 10902115).

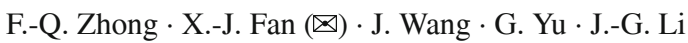

LHD, Institute of Mechanics,

Chinese Academy of Sciences,

100190 Beijing, China

e-mail: xfan@imech.ac.cn absorbed by the fuel is minimal, the hydrocarbon fuel would remain in the liquid state. As the flight speed increases and the fuel absorbs more heat, the fuel temperature may exceed its thermodynamic critical value, and the fuel becomes supercritical when its pressure is also supercritical. For China No. 3 aviation kerosene, the critical temperature and pressure are approximately $640 \mathrm{~K}$ and $2.4 \mathrm{MPa}$, respectively [4]. The supercritical state differs from the liquid or gas state due to many of its unique properties. It exhibits liquid-like density and gas-like diffusivity, zero latent heat and large compressibility $[3,5,6]$. During fuel injection, the supercritical fuel can be directly transformed to the gaseous state without atomization and vaporization processes. When the fuel temperature is sufficiently high, the fuel pyrolysis occurs as well [7]. Experimental results $[6,8]$ demonstrated that the overall burning intensity as well as the combustion efficiency improved with supercritical/cracked kerosene injection. However, further enhancement in combustion efficiency was limited by the shorter penetration depth of supercritical/cracked kerosene due to the lower density.

For gaseous fuel injection, the penetration depth could be increased with higher fuel/air pressure ratio. If the injection pressure is limited as in the case of scramjet applications, one alternative way to improve the penetration is to increase the injection Mach number of the fuel jet [9]. Our early studies $[6,8]$ demonstrated that supercritical/cracked kerosene could be accelerated to sonic speed using a sonic nozzle. However, for supercritical kerosene, its ratio of special heats is much closer to one, which results in a rapid fall in the fuel pressure and temperature during acceleration and two-phase flow could occur. For example, the China No. 3 kerosene with total temperature of approximately $650 \mathrm{~K}$ and total pressure of 4.0 MPa will start to condense when accelerated to the sonic speed. It remains unclear what a temperature and pressure range should be used to accelerate the supercritical kerosene to a given Mach number without any condensation. To simulate these acceleration processes of supercritical kerosene, a computational method was developed $[6,10]$ based on the principle of extended correspond- 
ing states (ECS) [11]. Isentropic assumption was used in the simulation for simplicity. A 3-species kerosene surrogate was used to calculate the thermophysical properties of China No. 3 kerosene during acceleration. The critical temperature and pressure at Mach one for supercritical kerosene have been calculated for varying stagnation conditions. Based on these calculations, the mass flow rate of supercritical kerosene through a sonic nozzle was obtained and validated experimentally $[6,10]$. To simulate the thermophysical properties of kerosene more accurately, a 10-species surrogate was developed later [12].

In this paper, the above computational method is further used to study the acceleration process of supercritical kerosene through a converging-diverging nozzle. The conditions for supercritical kerosene to reach a supersonic speed have been determined. Based on the calculation, an axissymmetric converging-diverging nozzle has been designed and the nozzle flow was investigated experimentally. The supercritical kerosene was prepared using a two-stage kerosene heating and delivery system $[6,8,12]$, which was capable of heating the kerosene up to a temperature of $820 \mathrm{~K}$ and a pressure of $5.5 \mathrm{MPa}$ with a maximum mass flow rate of $100 \mathrm{~g} / \mathrm{s}$. The minimum stagnation temperature of supercritical kerosene at given pressures to generate a stable supersonic flow was then determined and compared to the numerical results.

\section{Thermophysical properties of supercritical kerosene}

Kerosene is a complicated hydrocarbon mixture with thousands of species. In terms of volume, the China No. 3 aviation kerosene is approximately composed of 53\% alkanes, $39 \%$ cycloalkanes, $5 \%$ benzenes and $3 \%$ naphthalenes. Accordingly, a 10-species surrogate [12] was developed to determine its thermophysical properties based on the principle of extended corresponding states (ECS) [11]. Details of the surrogate are given in Table 1.

Table 1 The 10-component surrogate of China No. 3 kerosene

\begin{tabular}{lll}
\hline & Composition & Molar percentage/\% \\
\hline \multirow{4}{*}{ Alkanes } & n-Octane & 6 \\
& n-Decane & 10 \\
& n-Dodecane & 20 \\
& n-Tridecane & 8 \\
& n-Tetradecane & 10 \\
& n-Hexadecane & 10 \\
\hline \multirow{3}{*}{ Cycloalkanes } & Methylcyclohexane & 20 \\
& Trans-1, 3-Dimethyl- & 8 \\
& cyclopentane & \\
\hline Benzenes & Propylbenzene & 5 \\
\hline Naphthalenes & 1-Methylnaphthalene & 3 \\
\hline
\end{tabular}

Figure 1 is the calculated phase diagram of the 10 species kerosene surrogate, which shows the density as functions of temperature and pressure for various kerosene states. The measured kerosene densities at relatively low temperatures, reported previously [6], are also plotted in the figure for comparison. Under supercritical pressures $(\geq 2.4 \mathrm{MPa})$, as the fuel temperature rises and exceeds the critical value, kerosene in the liquid state will directly enter the supercritical region and no boiling occurs.

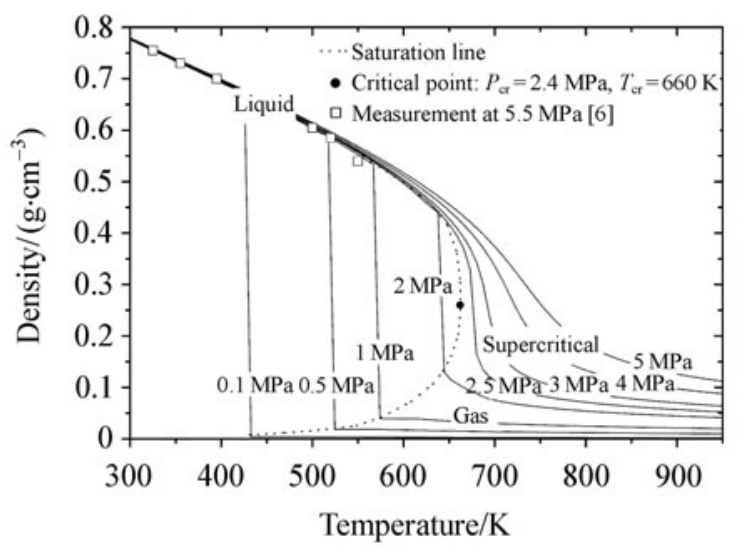

Fig. 1 Calculated density-temperature isobars for China No. 3 kerosene

Figures 2 and 3 show the variation of specific heat $C_{p}$ and the ratio of specific heats $\gamma$ as functions of temperature and pressure. Near the critical region, both $C_{p}$ and $\gamma$ change dramatically with temperature and their peak values are observed near the pseudo-critical temperature (approximately $700 \mathrm{~K}$ for $3 \mathrm{MPa}$ and $730 \mathrm{~K}$ for $5 \mathrm{MPa}$ ). Figure 4 shows the calculated speed of sound $a$ as functions of temperature and pressure. Large variations in $a$ are also found near the critical region, and for kerosene, no simple relationship such as $a=\sqrt{\gamma R T}$ could be found.

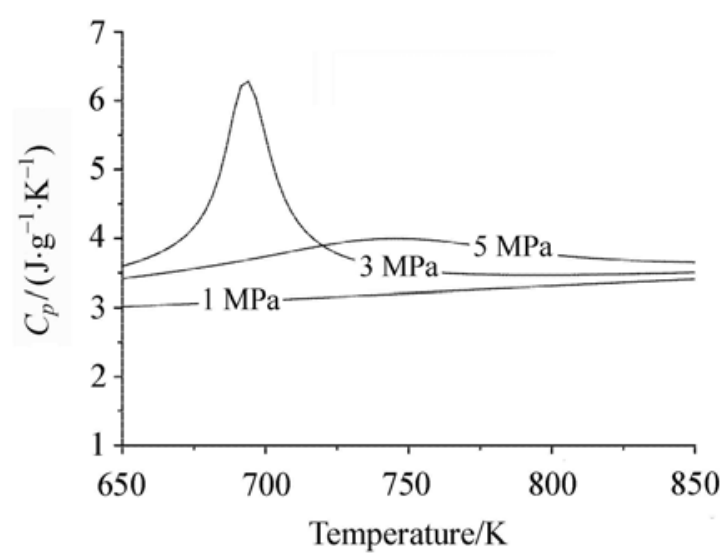

Fig. 2 Calculated specific heat for China No. 3 kerosene 


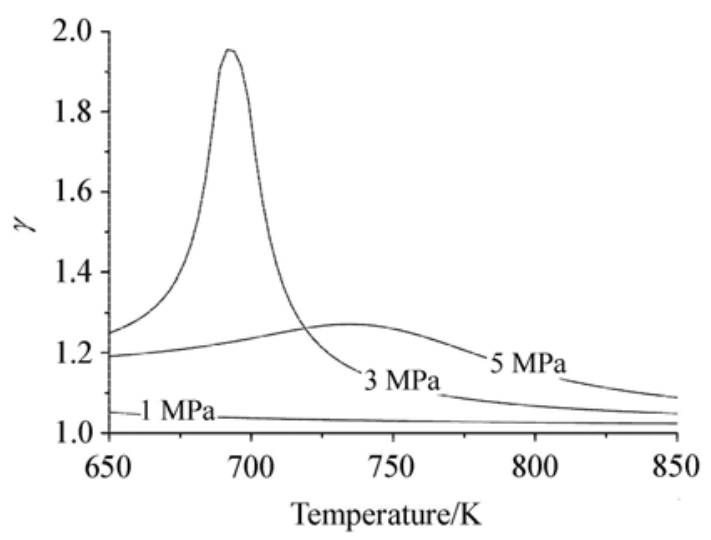

Fig. 3 Calculated ratio of specific heats for China No. 3 kerosene

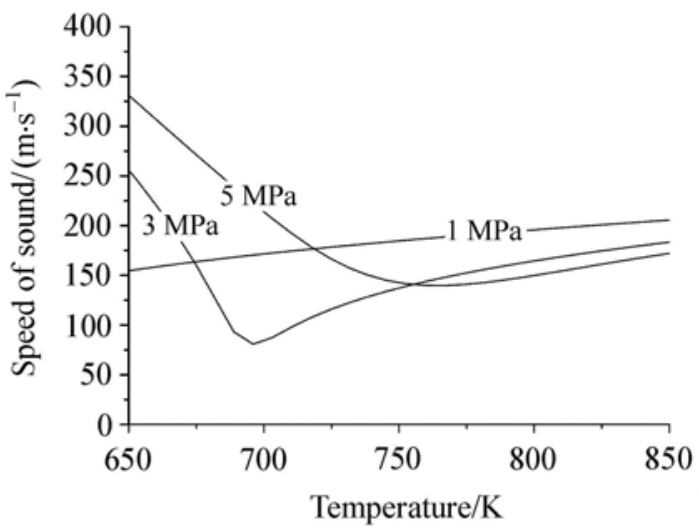

Fig. 4 Calculated speed of sound for China No. 3 kerosene

The following sections will show that the distinct thermophysical properties would remarkably affect the characteristics of compressible flow of kerosene.

\section{Numerical analysis of isentropic acceleration of kerosene}

With isentropic assumption, the flow properties can be determined using the following equations

$\rho u A=\dot{m}$,

$s_{0}=s_{0}\left(T_{0}, P_{0}\right)$,

$h+\frac{1}{2} u^{2}=h+\frac{1}{2}(M a)^{2}=h_{0}\left(T_{0}, P_{0}\right)$,

$P=P\left(s_{0}, T\right), \quad \rho=\rho\left(s_{0}, T\right), \quad h=T\left(s_{0}, T\right)$,

where $s$ and $h$ denote entropy and enthalpy, respectively; $M$ is the Mach number and $a$ is the speed of sound. Given the stagnation temperature $T_{0}$ and stagnation pressure $P_{0}$, the entropy $s_{0}$ can be calculated. Thus, the thermophysical properties of the kerosene, such as pressure, enthalpy at given $s_{0}$ and $T$, can be calculated using the 10 -species surrogate and the NIST Supertrapp [11]. Using Eqs. (1) and (3), the velocity and Mach number can be calculated along the isentropic line for varying cross-sectional area $A(x)$.

Figure 5 shows the isentropic acceleration lines of supercritical kerosene in the density phase diagram with a stagnation pressure of $5 \mathrm{MPa}$ and varying stagnation temperatures. It is found that as kerosene accelerates, its temperature and pressure decrease and it directly enters the gas-phase region. For the same stagnation pressure $P_{0}$, the decreasing stagnation temperature $T_{0}$ causes the isentropic lines to approach the two-phase dome region. Once the kerosene enters the two-phase region, i.e., condensation occurs, the acceleration would become unstable and the kerosene will not be able to accelerate to a supersonic flow. Figure 6 plots the isentropic lines with a higher stagnation pressure $\left(P_{0}=10 \mathrm{MPa}\right)$. As shown in the figure, for the same stagnation temperature, increasing $P_{0}$ causes the isentropic lines to be closer to the two-phase region, and consequently, makes the kerosene condensation much easier.

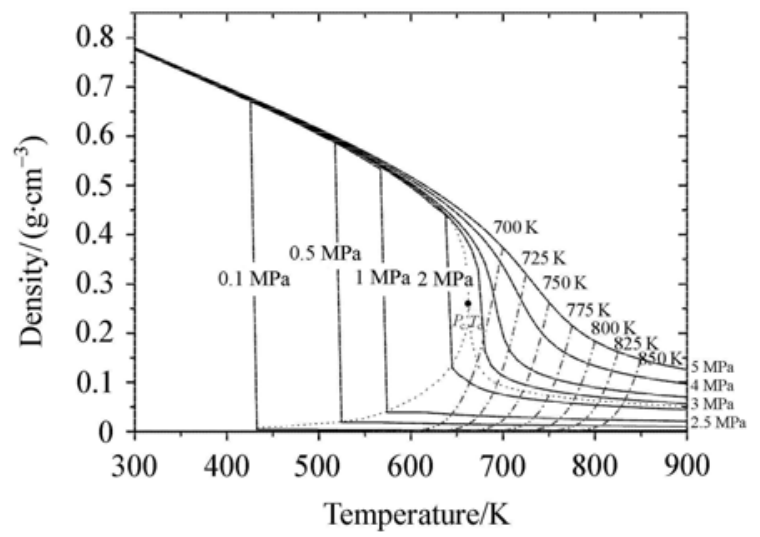

Fig. 5 Isentropic acceleration lines of supercritical kerosene with different stagnation temperatures and fixed stagnation pressure of $5 \mathrm{MPa}$

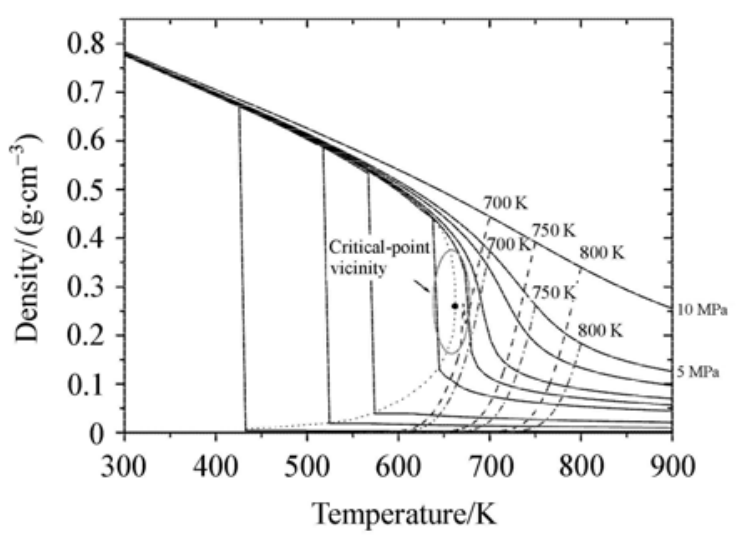

Fig. 6 Isentropic acceleration lines of supercritical kerosene with different stagnation temperatures and two fixed stagnation pressures of 5 and $10 \mathrm{MPa}$ 
Figure 7 shows the kerosene Mach number in the quasione-dimensional flow as a function of the cross-sectional area normalized by the throat area $A^{*}$. The $\frac{A}{A^{*}} \sim M$ relationship for ideal gas flow with $\gamma=1.4$ is also plotted for comparison. Significant difference between the two cases is observed in the supersonic flow region. Compared to the ideal gas flow with $\gamma=1.4$, a much larger area ratio is required for kerosene to reach the same Mach number, especially for Mach numbers greater than 1.5.

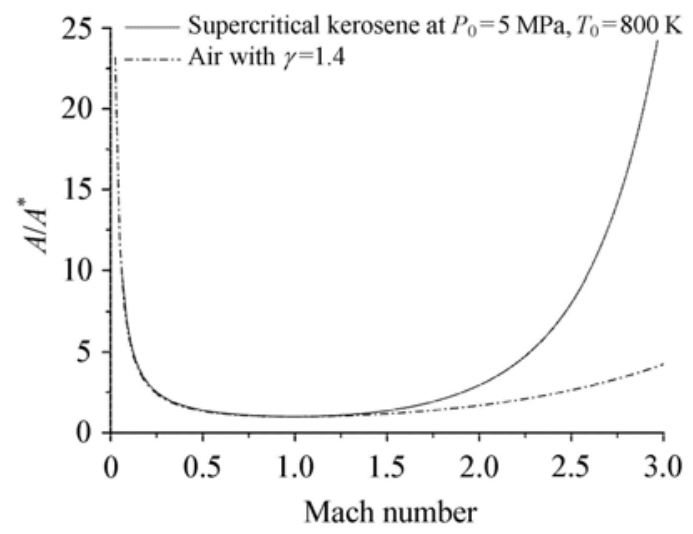

Fig. 7 Relationships of the area ratio and Mach number for 1-D kerosene and ideal gas flows

\section{Experimental and numerical studies of kerosene nozzle flow}

\subsection{Experimental set-up}

Experiments of kerosene flow through a convergingdiverging nozzle were conducted at varied stagnation temperatures and pressures. Figure 8 shows the sketch of the nozzle and the locations of wall pressure taps. Figure 9 shows the photograph of the nozzle assembly. The tested kerosene was China No. 3 aviation kerosene, for which a surrogate was used in calculation correspondingly.

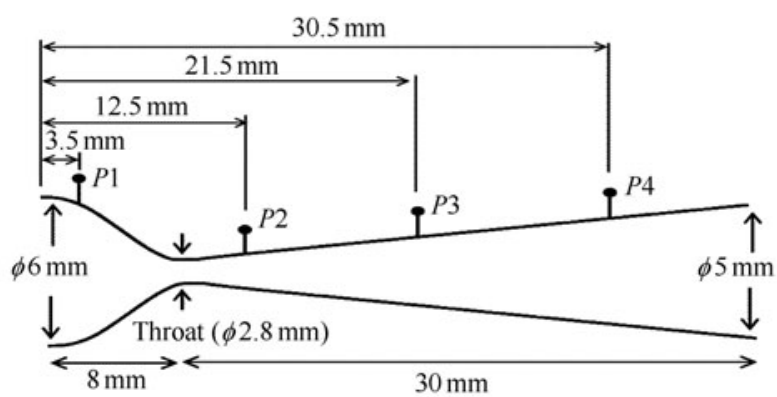

Fig. 8 Sketch of the converging-diverging nozzle and locations of the pressure taps

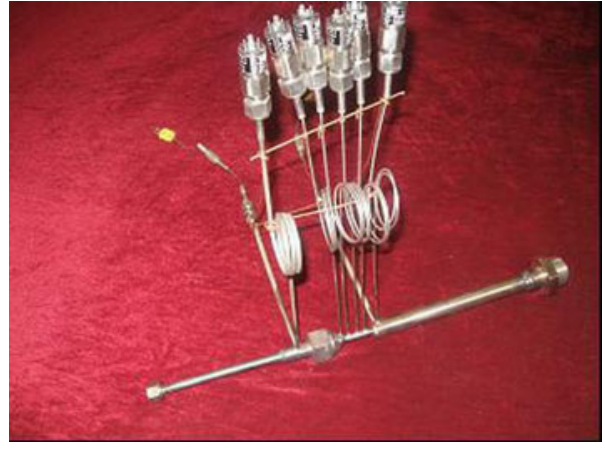

Fig. 9 Photograph of the converging-diverging nozzle and measurement probes

As indicated in Fig. 8, the stagnation temperature and pressure were measured upstream the nozzle throat with a type- $\mathrm{K}$ thermocouple and fast-response pressure transducer, respectively. Downstream of the throat, three holes of $\phi 0.5 \mathrm{~mm}$ were drilled in the wall for the static pressure measurements (P2-P4). A two-stage kerosene heating system was used to prepare the supercritical kerosene at desired temperatures of $650 \mathrm{~K}-820 \mathrm{~K}$ and pressures of $3-5.5 \mathrm{MPa}$ with mass flow rates of $20-100 \mathrm{~g} / \mathrm{s}$. The pre-heated kerosene was driven by pressurized nitrogen at given pressures (3$5.5 \mathrm{MPa}$ ) and flew through the nozzle section with two pneumatic valves controlled by a computer. To establish a sufficient pressure gradient for a supersonic flow, the exit of the nozzle was connected to a $3 \mathrm{~m}$ long pipe with an inner diameter of $26 \mathrm{~mm}$. The fuel mass flow rate was calibrated and measured using a fuel collection system. Details of the fuel heating, control and collection system were described in our previous work $[6,7,12]$.

The outside of the nozzle was well insulated with asbestos and the nozzle's length-to-diameter ratio is relatively small. Therefore, the heat and frictional losses are negligible and will not be considered in data reduction.

4.2 Experimental results and comparison with numerical calculations

Figure 10 shows the time history of the static pressure at the four locations (P1-P4) as indicated in Fig. 8 with the stagnation temperature of $805 \mathrm{~K}$ and stagnation pressure of $5.2 \mathrm{MPa}$. The kerosene flow was started at $t=6 \mathrm{~s}$. Figure 10 shows that all the pressure values become nearly constant (except the trivial change caused by the accuracy of the pressure transducer) for $t>9.5 \mathrm{~s}$, indicating a steady flow through the nozzle. Figure 11 shows the static pressure distribution along the nozzle measured at $t=12 \mathrm{~s}$. The numerical result is also plotted in the figure for comparison. As the flow accelerates, the fuel pressure decreases rapidly especially downstream the throat. The pressure decreased to only $0.67 \mathrm{MPa}$ at $x=31 \mathrm{~mm}$. Figure 11 indicates that a supersonic flow established downstream of the nozzle throat and it also shows that the measurements agree well with the numerical predictions. 


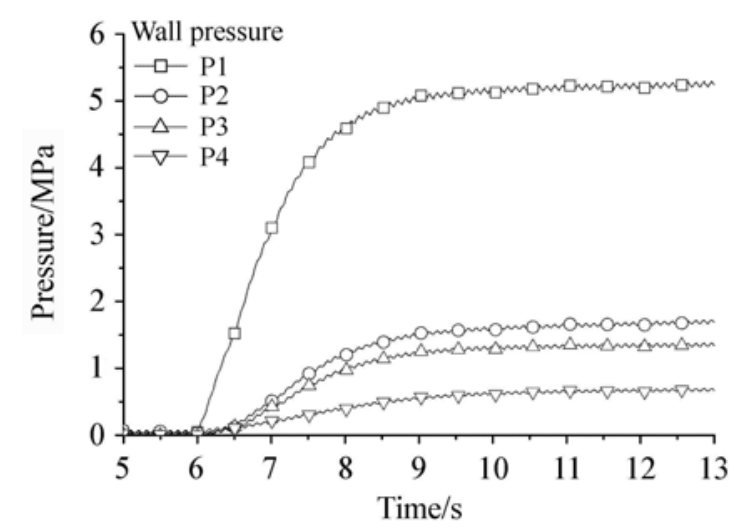

Fig. 10 Time histories of wall pressures P1-P4 of the nozzle

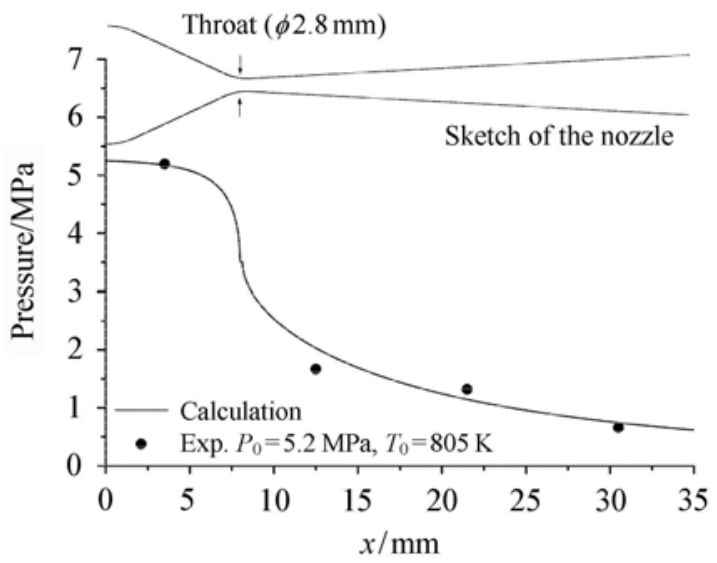

Fig. 11 Comparison of the measured wall pressure with calculation

Figure 12 shows the distributions of the Mach number and static temperature along the nozzle by numerical analysis for the same conditions shown as in Fig. 11. The exit Mach number is approximately 2.05. Because the $\gamma$ of the

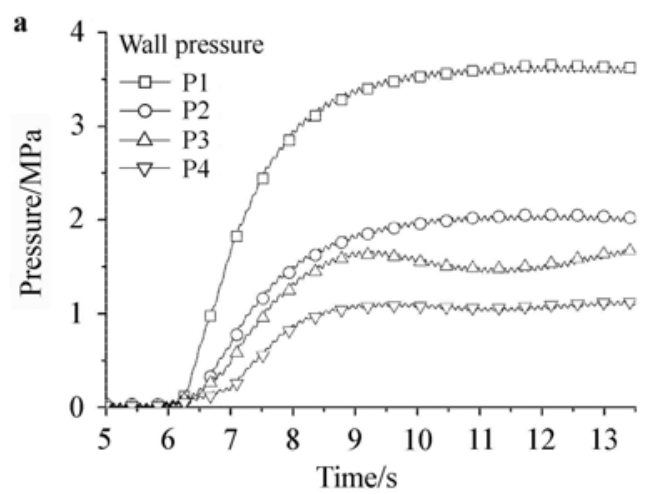

kerosene is close to 1.0 , the exit temperature decreases by only $50 \mathrm{~K}$ to approximately $756 \mathrm{~K}$.

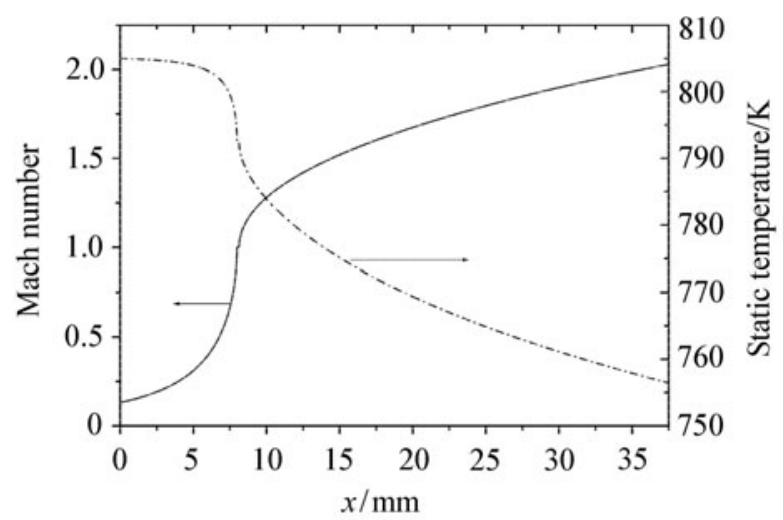

Fig. 12 Calculated distributions of static temperature and Mach number along the nozzle

4.3 Experimental results at varying stagnation temperatures and pressures

Figures $13 \mathrm{a}$ and $13 \mathrm{~b}$ show the time histories of the measured static pressure for two different combinations of stagnation temperature and pressure: (a) $680 \mathrm{~K} / 3.5 \mathrm{MPa}$ and (b) $680 \mathrm{~K} / 4.6 \mathrm{MPa}$. Because of the relatively low stagnation temperature, as shown in Fig. 13a, the static pressures in Case (a) became unstable during the experiment, indicating some transitional process, such as local condensation, occurred in the flow. For the Case (b) with the same stagnation temperature but a higher pressure, Fig. 13b shows that all pressures downstream the throat decreased to very low values, which indicates that no supersonic flow was established inside the nozzle.

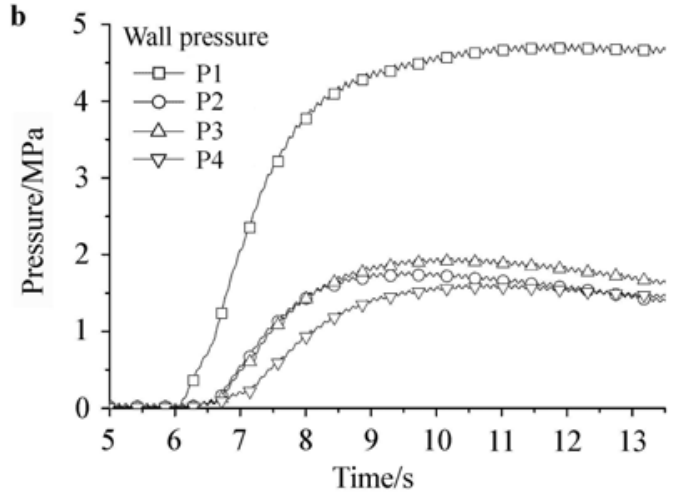

Fig. 13 Time histories of the measured wall pressures for two cases with low inlet temperatures. a $T_{0} / P_{0}: 680 \mathrm{~K} / 3.5 \mathrm{MPa}$; b $T_{0} / P_{0}$ : $680 \mathrm{~K} / 4.6 \mathrm{MPa}$

Table 2 summarizes the experimental results with different stagnation temperatures and pressures. It is found that no stable supersonic flow of supercritical kerosene could be established inside the converging-diverging nozzle for stagnation temperatures lower than approximately $700 \mathrm{~K}$ no matter what pressure is applied. The kerosene with a relatively 
low stagnation temperature will start to condense and enter the two-phase region during the isentropic acceleration, which is consistent with the numerical analysis given in Sect. 3. Hence, based on the present study, the minimum stagnation temperature for supercritical kerosene to establish a supersonic flow is approximately $730 \mathrm{~K}$ and the corresponding non-dimensional temperature normalized by the critical value is approximately 1.1. Because significant thermal cracking will occur for fuel temperatures higher than approximately $820 \mathrm{~K}[7,8]$, the temperature range for generating a stable supersonic flow is quite narrow.

Table 2 Summary of the nozzle flow experiments of supercritical kerosene

\begin{tabular}{llll}
\hline Test No. & $P_{0} / \mathrm{MPa}$ & $T_{0} / \mathrm{K}$ & Supersonic flow \\
\hline 1 & 3.5 & 800 & Yes \\
2 & 3.4 & 740 & Yes \\
3 & 3.5 & 680 & unstable \\
4 & 3.1 & 670 & unstable \\
5 & 4.6 & 790 & Yes \\
6 & 4.6 & 730 & Yes \\
7 & 4.5 & 700 & unstable \\
8 & 4.6 & 680 & No \\
9 & 5.2 & 805 & Yes \\
10 & 5.1 & 750 & Yes \\
\hline
\end{tabular}

\section{Conclusions}

In this paper, the compressible flow of supercritical kerosene has been studied both numerically and experimentally. Isentropic relations of the kerosene flow have been analyzed and compared with those of ideal gas. Based on the calculation, a converging-diverging nozzle has been designed and tested. Static pressures along the nozzle for supercritical kerosene with different stagnation temperatures and pressures were measured and the results agree with the numerical predictions.

Due to the complicated thermophysical properties of supercritical kerosene flow, its isentropic relations, such as the area ratio vs. Mach number, temperature and pressure drop vs. Mach number, are significantly different from those of ideal gas.

Supercritical kerosene with properly chosen stagnation temperatures can be accelerated to a stable supersonic flow without fuel condensation. For China No. 3 kerosene, this temperature range is $730 \mathrm{~K}-820 \mathrm{~K}$. Decreasing the temperature or increasing the pressure causes the isentropic lines to approach the critical region and the kerosene becomes more likely to enter the two-phase region. In some cases of the experiment, fuel condensation will occur and no supersonic flow can be established.

It is noteworthy that in a realistic cooling system with high wall heat flux or small flow channels, the heating and frictional effects will become significant. Further research of the compressible kerosene flow with heating effect (Rayleigh flow) and friction effect (Fanno flow) is imperative.

Acknowledgment The authors thank Ying Li, Xuesong Wei, and Peng Huang for their technical support.

\section{References}

1 Huang, H., Spadaccini, L.J., Sobel, D.R.: Fuel-cooled thermal management for advanced aero-engines. ASME Journal of Engineering for Gas Turbines and Power 126, 284-293 (2004)

2 Sobel, D.R., Spadaccini, L.J.: Hydrocarbon fuel cooling technologies for advanced propulsion. ASME Journal of Engineering for Gas Turbines and Power 119, 344-351 (1997)

3 Yang, V.: Modeling of supercritical vaporization, mixing and combustion processes in liquid-fueled propulsion system. Proceeding of the Combustion Institute 28, 925-942 (2000)

4 Sun, Q.M., Mi, Z.T., Zhang, X.W.: Determination of critical properties $\left(T_{c}, P_{c}\right)$ of endothermic hydrocarbon fuels RP-3 and simulated JP-7. Chinese Journal of Fuel Chemistry and Technology 34(4), 466-470 (2006) (in Chinese)

5 Edwards, T., Maurice, L.Q.: Surrogate mixtures to represent complex aviation and rocket fuels. Journal of Propulsion and Power 17, 461-466 (2001)

6 Fan, X.J., Yu, G., Li, J.G., et al.: Investigation of vaporized kerosene injection and combustion in a supersonic model combustor. Journal of Propulsion and Power 22(1), 103-110 (2006)

7 Zhong, F.Q., Fan, X.J., Yu, G., et al.: Thermal cracking of aviation kerosene for scramjet applications. Science in China, Series E 52(9), 2644-2652 (2009)

8 Fan, X.J., Yu, G., Li, J.G., et al.: Combustion and ignition of thermal cracked kerosene in supersonic model combustors. Journal of Propulsion and Power 23(2), 317-324 (2007)

9 Billig, F.S., Orth, R.C., Lasky, M.: A unified analysis of gaseous jet penetration. AIAA Journal 9(6), (1971)

10 Fan, X.J., Yu, G.: Analysis of thermophysical properties of daqing RP-3 aviation kerosene. Journal of Propulsion Technology 27(2), 187-192 (2006) (in Chinese)

11 Ely, J.F., Huber, M.L.: NIST standard reference database 4-nist thermophysical properties of hydrocarbon mixtures. National Inst. of Standards, Gaithersburg, MD, Feb. (1990)

12 Zhong, F.Q., Fan, X.J., Yu, G., et al.: Heat transfer of aviation kerosene at supercritical conditions. Journal of Thermophysics and Heat Transfer 23(3), 543-550 (2009) 